\title{
Deutscher Bibliotheksverband beteiligt sich am „Kultur macht stark“-Sommer des Bundesbildungsministeriums
}

\author{
https://doi.org/10.1515/bd-2021-0066
}

Mit zusätzlichen Ferienangeboten in allen Bundesländern will das Förderprogramm „Kultur macht stark: Bündnisse für Bildung“ einen Beitrag zu mehr Bildungsgerechtigkeit und Teilhabe leisten. Bundesbildungsministerin Anja Karliczek hat dafür den „Kultur macht stark“-Sommer ins Leben gerufen.

Als langjähriger Programmpartner des Bundesministeriums für Bildung und Forschung (BMBF) beteiligt sich der Deutsche Bibliotheksverband e.V. (dbv) mit seinem Projekt „Total Digital! Lesen und erzählen mit digitalen Medien“ an der Initiative. Für diese konzertierte Aktion für mehr kulturelle Bildungsangebote in den Ferien wurden die Förderbedingungen angepasst, so dass nun auch überregionale Einrichtungen - wie z.B. eine Bibliotheksfachstelle - mit zwei lokalen Partnern ein Bündnis bilden kann.

Kinder, die unter schwierigen sozialen Bedingungen aufwachsen, sind durch die Corona-Pandemie besonders betroffen. Durch diese Kulturangebote wird ihnen der Zugang zu Bildung erleichtert. Über „Total Digital!“ werden Projekte der digitalen Leseförderung in Zusammenarbeit mit einer Bibliothek zu 100 Prozent gefördert.

Weitere Informationen zum Programm „Total Digital!“ sowie zu kontaktlosen, kontaktarmen oder Präsenz-Projektbeispielen finden Sie unter: https://www. lesen-und-digitale-medien.de/de_DE/best-practice-projekte-corona.

\section{„Total Digital! Lesen und erzählen mit digitalen Medien“}

Der dbv beteiligt sich seit 2013 am Förderprogramm „Kultur macht stark. Bündnisse für Bildung “ des Bundesministeriums für Bildung und Forschung (BMBF). In der ersten Programmphase von 2013 bis 2017 mit dem Projekt „Lesen macht stark: Lesen und digitale Medien“, seit 2018 mit dem Projekt „Total Digital!“. Das Förderprogramm richtet sich in erster Linie an Kinder und Jugendliche von drei bis achtzehn Jahre, denen der Zugang zu Bildung erschwert ist. Mit „Kultur macht stark“ fördert das BMBF auch in der zweiten Programmphase (2018 bis 2022) außerschulische Projekte der Kulturellen Bildung für mehr gesellschaftliche Teilhabe und Chancengleichheit mit 250 Millionen Euro. Neben dem dbv sind 28 weitere Verbände und Initiativen Programmpartner von „Kultur macht stark“.

Mehr Informationen finden Sie unter www.lesen-und-digitale-medien.de. 


\section{Der Deutsche Bibliotheksverband e.V. (dbv)}

Der Deutsche Bibliotheksverband e.V. (dbv) vertritt mit seinen fast 2.100 Mitgliedern bundesweit über 9.000 Bibliotheken mit 25.000 Beschäftigten und elf Millionen Nutzer^innen. Sein zentrales Anliegen ist es, Bibliotheken zu stärken, damit sie allen Bürger*innen freien Zugang zu Informationen ermöglichen. Der Verband setzt sich ein für die Entwicklung innovativer Bibliotheksleistungen für Wissenschaft und Gesellschaft. Als politische Interessensvertretung unterstützt der dbv die Bibliotheken insbesondere auf den Feldern Informationskompetenz und Medienbildung, Leseförderung und bei der Ermöglichung kultureller und gesellschaftlicher Teilhabe für alle Bürger`innen.

Mehr Informationen finden Sie unter www.bibliotheksverband.de.

\section{Kontakte Deutscher Bibliotheksverband e.V. (dbv)}

Brigitta Wühr

Projektleiterin „Total Digital!“

Tel.: + 49 (0)30 644989913 | E-Mail: wuehr@bibliotheksverband.de

Kristin Bäßler

Leitung Kommunikation / Pressesprecherin

Tel.: + 49 (0)30 6449899 25 | E-Mail: baessler@bibliotheksverband.de 